\title{
Synthesis of a Lever-Cam Motion Transducer for a Rotary Vane Pump
}

\author{
Andrey Perminov \\ Department of Electric Coupling and \\ Automation Systems \\ Pskov State University \\ Pskov, Russia \\ alp-mail@mail.ru \\ Alexander Khitrov \\ Department of Electric Coupling and \\ Automation Systems \\ Pskov State University \\ Pskov, Russia \\ khitrov-pscov@mail.ru
}

\author{
Alexander Ilyin \\ Department of Electric Coupling and \\ Automation Systems \\ Pskov State University \\ Pskov, Russia \\ al.ilyin@yandex.ru
}

\author{
Sergey Tikhonov \\ Department of Automobile Transport \\ Pskov State University \\ Pskov, Russia \\ sit42@rambler.ru
}

\begin{abstract}
The article solves problem of synthesizing a lever-cam motion transducer that converts rotary motion of input shaft of a rotary pump into motion of its vanes required for volumetric pumping of gases and liquids. Analytical expressions for theoretical profile of cam of lever-cam transducer of the movement of rotary vane pump are obtained. The cam profile was built according to analytical expressions.
\end{abstract}

Keywords - rotary vane pump, lever-cam motion transducer, volumetric pumping gas.

\section{INTRODUCTION}

The creation of a pump that provides volumetric pumping of a working fluid regardless of the working fluid type (gas, liquid and gas-liquid mixture) that enters the pump inlet is very important for a number of industries (gas, oil, pharmaceutical, etc.)

An alternative to existing types of pumps with volumetric pumping is a rotary vane pump with a levercam motion converter [1] - [3].

Structurally pump consists of a rotary vane group and a lever-cam motion transducer (Fig. 1).

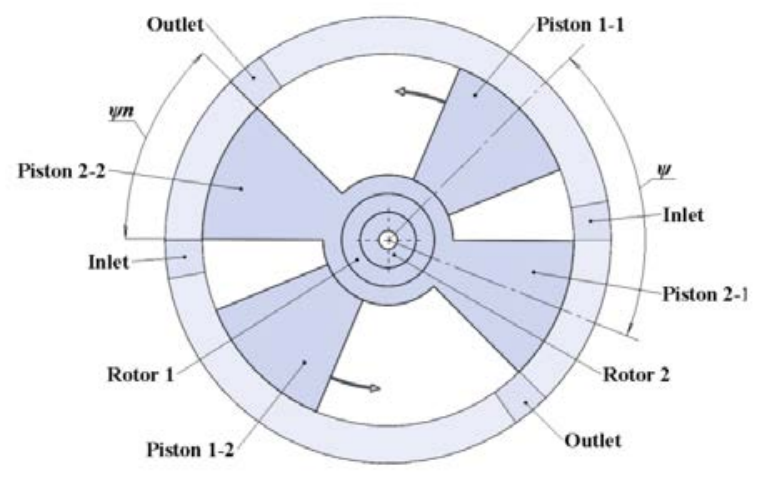

Fig. 1. Rotary vane pump design.

The rotor-vane group consists of a body and two coaxial rotors, each of which carries a vane of two diametrically located pistons. Pistons have an angular dimension $\psi_{n}$. Body has two inlet and two outlet ports. Inter-piston space contains four chambers of variable volume. Opening and closing of windows is done by respective piston surfaces [4], [5].

Pump works as follows. Volume of chamber opposite open inlet window increases to a maximum value and is filled with a working fluid. Then, this volume of working fluid with closed inlet port is transferred to outlet zone, and with open outlet port, release occurs with a decrease in chamber volume to a minimum value. 
On one revolution of output shaft, each chamber makes two releases of working fluid and, therefore, with four chambers, pump makes eight releases. Moreover, pumping process is really voluminous, i.e. it is volume that is pumped, regardless of whether gas or liquid fills its volume when entering chamber.

The type of working fluid affects only power of drive motor.

\section{ORGANIZATION OF MOVEMENT}

To organize motion of vanes described above, it is proposed to use a lever-cam motion transducer. A similar mechanism was first proposed in patent RU 2374526 [1] for a rotary vane engine with external heat supply [2]. The kinematic diagram of the mechanism is shown in Fig. 2.

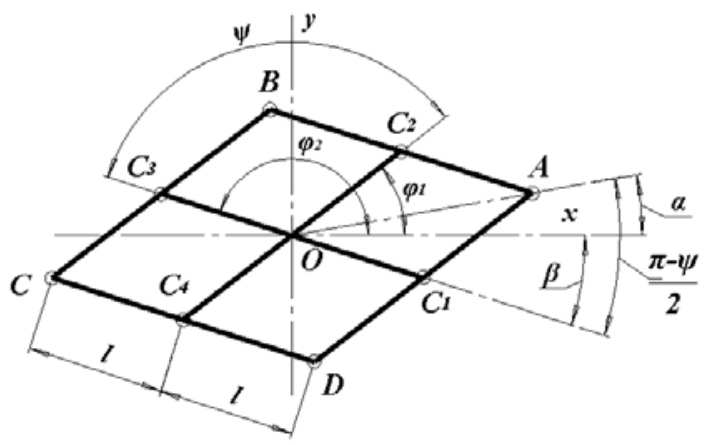

Fig. 2. Kinematic diagram of mechanism.

Four links $A B, B C, C D$ and $D A$ of length $2 l$ each are pivotally connected to each other and form a rhomboid. Fork $C_{1} C_{3}$ of vane 1 and fork $C_{2} C_{4}$ of vane 2 are pivotally attached to centers of links of rhomboid. Axes of vanes have angular coordinates $\varphi_{1}$ and $\varphi_{2}$, respectively.

Rhomboid vertices $A, B, C$, and $D$ are equipped with rollers that roll on cam. A cam profile is such that it makes the axes of rollers $\mathrm{A}, \mathrm{B}, \mathrm{C}$ and $\mathrm{D}$ move along so-called theoretical cam profile, which sets law of motion of vanes. Profile of the cam itself is equidistant (internal or external) to theoretical profile. The cam used in rotary vane pump has a theoretical profile in polar coordinates $\rho$ and $\alpha$ of form

$$
\rho(\alpha)=2 l \sin (a+b \cos (2 \alpha)),
$$

here $a=\pi / 4, b=\pi / 4-\psi_{\min } / 2$. Profile (1) provides a change in the angle between axes of vanes according to the harmonic law $\psi(\alpha)=2 a+2 b \cos (2 \alpha)$, which cannot be used for pump under consideration [6], [7].

Taking this circumstance into account, we formulate the problem as follows. It is required to synthesize a cam profile that would ensure that one vane stops, while other blade would move at which the angle $\psi$ between axes of vanes either decreases from value $\psi_{\max }$ to $\psi_{\min }$ (outlet), or increases from $\psi_{\min }$ to $\psi_{\max }$ (inlet), and also the movement of the inlet isolated volume to the outlet/inlet area.

\section{GEOMETRIC RELATIONSHIPS}

The configuration of rhomboid is completely determined by links length $2 l$ and the angles $\varphi_{1}$ and $\varphi_{2}$ of vanes axes $C_{1} C_{3}$ and $C_{2} C_{4}$ with the $x$-axis. These angles are input variables of four-link structure. For output parameters we take length of half-diagonal $O A=\rho$ (polar radius) and angle $\alpha$ between $O A$ and $x$-axis (polar angle). Obviously, function $\rho(\alpha)$ defines theoretical cam profile.

An important geometric parameter is angle $\psi=\varphi_{1}$ $\varphi_{2}$ between vanes axes, which can vary within limits $\psi_{\min } \leq \psi \leq \psi_{\max }, \psi_{\max }=\pi-\psi_{\min }$, where value $\psi_{\text {min }}$ is set for design reasons.

From Fig. 2 we have

$$
\begin{gathered}
\beta=\pi-\psi-\varphi_{2}, \\
\alpha=\frac{\pi-\psi}{2}-\beta=\frac{\varphi_{1}+\varphi_{2}}{2}-\frac{\pi}{2}, \\
\rho=2 l \cos \left(\frac{\pi-\psi}{2}\right)=2 l \sin \left(\frac{\psi}{2}\right),
\end{gathered}
$$

whence next geometric relations follow:

$$
\begin{gathered}
\psi=\varphi_{1}-\varphi_{2}, \\
\varphi_{1}+\varphi_{2}=2 \alpha+\pi, \\
\varphi_{1}=\alpha+\frac{\psi}{2}+\frac{\pi}{2}, \varphi_{2}=\alpha-\frac{\psi}{2}+\frac{\pi}{2}, \\
\rho(\alpha)=2 l \sin \left(\frac{\psi}{2}\right), \bar{\rho}(\alpha)=\frac{\rho(\alpha)}{2 l}=\sin \left(\frac{\psi}{2}\right),
\end{gathered}
$$

where $\bar{\rho}$ is dimensionless polar radius.

\section{FIRST STAGE}

First stage begins from position $\alpha=0$, in which angle between axes of vanes $\psi=\psi_{\text {max }}$, and the angles $\varphi_{1}$ and $\varphi_{2}$, according to (5), have the following values:

$$
\begin{array}{r}
\varphi_{1}=\varphi_{10}=\frac{\psi_{\max }}{2}+\frac{\pi}{2}, \\
\varphi_{2}=\varphi_{20}=-\frac{\psi_{\max }}{2}+\frac{\pi}{2} .
\end{array}
$$

At this stage, the vane 1 must remain stationary, so angle $\varphi_{1}$ must have a constant value $\varphi_{10}$. Vane should move to approach vane 1 and according to (4), the angle $\varphi_{2}(\alpha)=2 \alpha+\pi-\varphi_{10}=2 \alpha-\psi_{\max } / 2+\pi / 2$. In this case, angle $\psi$ changes according to law

$$
\psi(\alpha)=\varphi_{10}-\varphi_{2}(\alpha)=\psi_{\max }-2 \alpha .
$$

Starting stage ends when angle $\psi$ reaches value $\psi_{\text {min }}$. Value of angle $\alpha$, at which this stage ends, will be denoted by $\alpha^{*}$ and found from relation (8):

$$
\alpha^{*}=\frac{\pi}{2}-\psi_{\min } .
$$

Vertex of rhomboid A in this segment moves along trajectory

$$
\bar{\rho}_{A}(\alpha)=\sin \left(\frac{\psi_{\max }}{2}-\alpha\right), 0 \leq \alpha \leq \alpha^{*} .
$$

and at the same time $\alpha=\alpha^{*}$ will take the position $A^{*}$. Thus, the polar radius $\bar{\rho}_{A}$, which at $\alpha=0$ has largest value 
$\bar{\rho}_{A}(0)=\sin \left(\frac{\psi_{\max }}{2}\right)=\bar{\rho}_{\max }$ decreases with increasing $\alpha$, and at $\alpha=\alpha^{*}$ takes smallest value $\bar{\rho}_{A}\left(\alpha^{*}\right)=$ $\sin \left(\frac{\psi_{\min }}{2}\right)=\bar{\rho}_{\text {min }}$.

The vertex of rhomboid $B$ corresponds to the polar radius $\bar{\rho}_{B}$, which is related to the radius $\bar{\rho}_{A}$; the obvious relation is $\bar{\rho}_{A}^{2}+\bar{\rho}_{B}^{2}=1$, which implies $\bar{\rho}_{B}(0)=$ $\cos \left(\frac{\psi_{\max }}{2}-\alpha_{A}\right)$. Considering that $\alpha_{A}=\alpha_{B}-\pi / 2$, we obtain the equation of the cam profile in the $B B^{*}$ section:

$$
\bar{\rho}_{B}(\alpha)=\sin \left(\alpha-\frac{\psi_{\max }}{2}\right), \frac{\pi}{2} \leq \alpha \leq\left(\alpha^{*}+\frac{\pi}{2}\right) .
$$

Polar radius $\bar{\rho}_{B}$, which at $\alpha=\pi / 2$ has smallest value $\bar{\rho}_{\text {min }}=\sin \left(\frac{\psi_{\min }}{2}\right)$, at $\alpha=\pi / 2+\alpha^{*}$ takes largest value $\bar{\rho}_{\text {max }}=\sin \left(\frac{\psi_{\text {max }}}{2}\right)$, (Fig. 3).

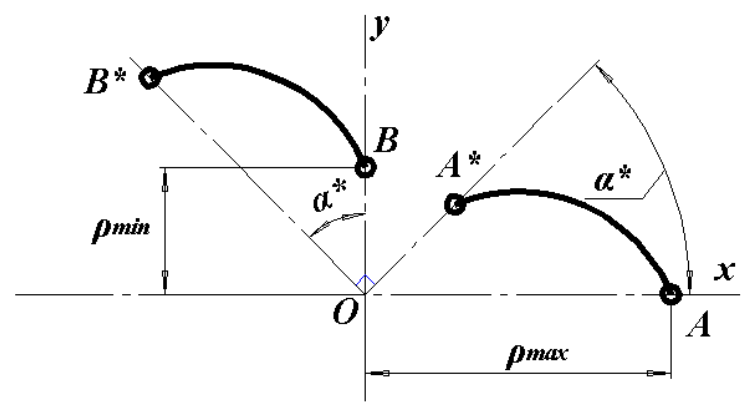

Fig. 3. First stage.

At the end of the starting stage, the working fluid is displaced in one pair of diametrically located chambers, and filling occurs in the other pair of chambers.

\section{SECOND STAGE}

At this stage, all four chambers are moved without changing volume: one pair of chambers to filling zone, other to discharge zone. Therefore, points $A^{*}$ and $B$ must be connected by an arc of a circle of radius $\bar{\rho}_{\text {min }}$, and points $B^{*}$ and $C$ - by an arc of a circle of radius $\bar{\rho}_{\max }$, with center of both circles at point $O$ (Fig. 4). We have:

$$
\begin{gathered}
\bar{\rho}(\alpha)=\bar{\rho}_{\text {min }}=\sin \left(\frac{\psi_{\min }}{2}\right)=\text { const, } \alpha^{*} \leq \alpha \leq \frac{\pi}{2} ; \\
\bar{\rho}(\alpha)=\bar{\rho}_{\max }=\sin \left(\frac{\psi_{\max }}{2}\right)=\mathrm{const}, \\
\left(\alpha^{*}+\frac{\pi}{2}\right) \leq \alpha \leq \pi .
\end{gathered}
$$

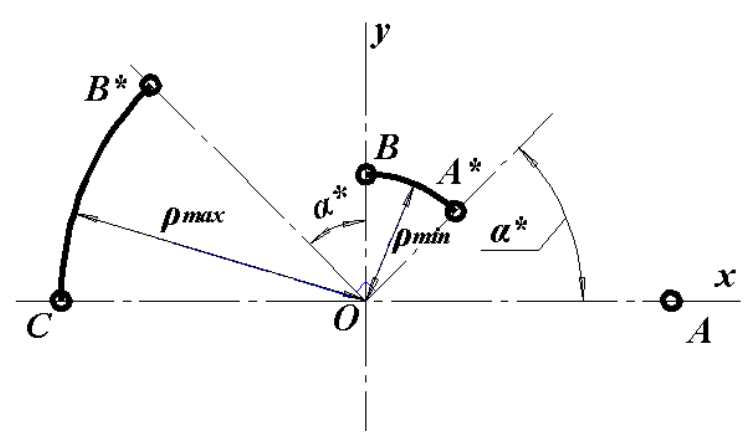

Fig. 4. Second stage.

\section{CAM PROFILE EQUATIONS IN POLAR COORDINATES}

In the range $0 \leq \alpha \leq \pi$, the cam profile is described by equations (10) - (12). The function $\bar{\rho}(\alpha)$ has period $\pi$, therefore, in the interval $\pi \leq \alpha \leq 2 \pi$, we have

$$
\begin{gathered}
\bar{\rho}(\alpha)=\sin \left(\alpha-\frac{\psi_{\max }}{2}\right), \pi \leq \alpha<\left(\alpha^{*}+\pi\right) ; \\
\bar{\rho}(\alpha)=\bar{\rho}_{\text {min }}=\sin \left(\frac{\psi_{\min }}{2}\right),\left(\alpha^{*}+\pi\right) \leq \alpha<\frac{3 \pi}{2} ; \\
\bar{\rho}(\alpha)=\sin \left(\frac{\psi_{\max }}{2}-\alpha\right), \frac{3 \pi}{2} \leq \alpha<\left(\alpha^{*}+\frac{3 \pi}{2}\right) ; \\
\bar{\rho}(\alpha)=\bar{\rho}_{\text {max }}=\sin \left(\frac{\psi_{\max }}{2}\right),\left(\alpha^{*}+\frac{3 \pi}{2}\right) \leq \alpha \leq 2 \pi .
\end{gathered}
$$

Equations (10) - (13) can be used to construct a theoretical cam profile. The theoretical profile for $\psi_{\min }=$ $45^{\circ}$ is shown in Fig. 5. Profile is built in relative units.

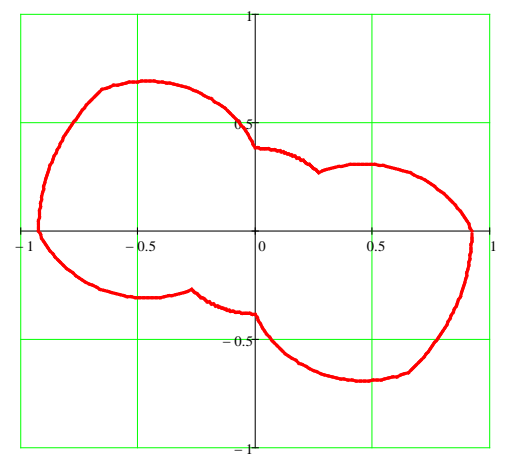

Fig. 5. Theoretical profile.

Using an equidistant offset from theoretical profile, actual cam profile is obtained. The appearance of cam is shown in Fig. 6. 


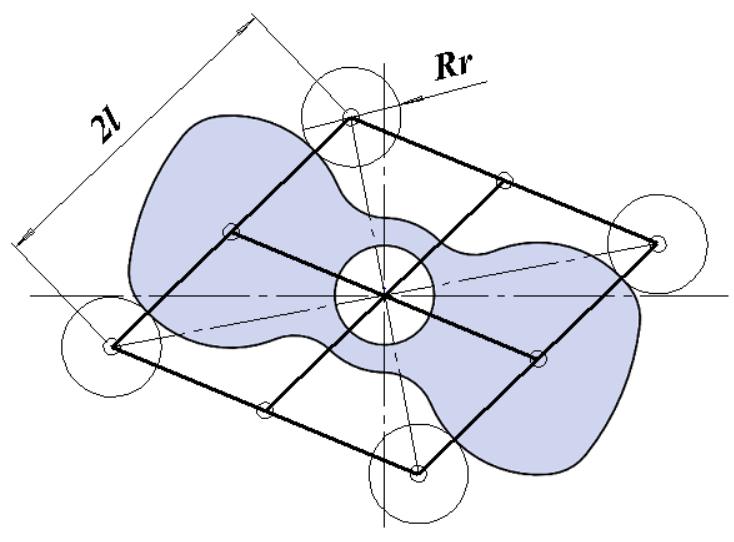

Fig. 6. Cam profile.

Angle between axes of vanes $\psi$ changes according to law shown in Fig. 7. As can be seen from the graph, synthesized profile ensures that one vane stops, while other vane moves at which angle $\psi$ between axes of vanes increases from $\psi_{\min }$ to $\psi_{\max }$ (inlet), and decreases from $\psi_{\max }$ to $\psi_{\min }$ (outlet). Also, the inlet isolated volume moves to the outlet/inlet zone.

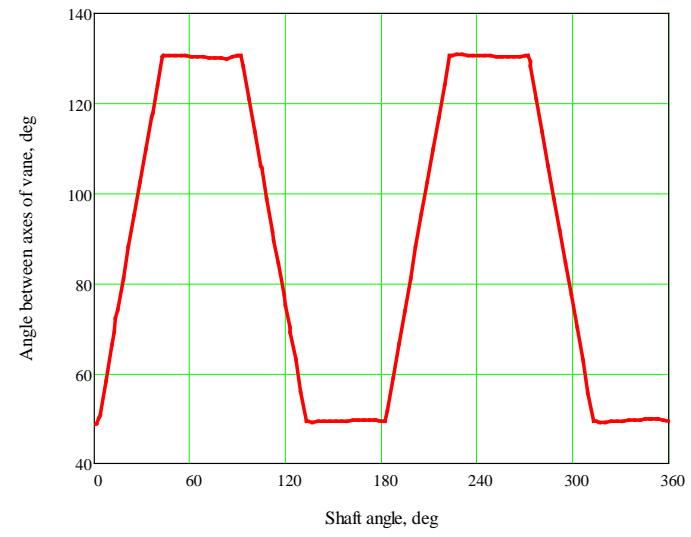

Fig. 7. Angle between axes of vanes $\psi$.

\section{CONCLUSION}

In this article, analytical expressions are obtained for theoretical profile of cam of lever-cam transducer of movement of a rotary vane pump. Cam profile was built according to analytical expressions.

Results of article can be used in design and manufacture of rotary vane pumps that provide volumetric pumping of both gas and liquid working bodies , as well as other machines of a similar type [8] - [11].

\section{REFERENCES}

[1] RF Patent 2374526 for an invention. Int. Cl. F16H25/04. Mechanism for converting motion / Y. N. Lukyanov, Y. N. Zhuravlev et al. Publ. 27.11.2009. Bull. Number 33.

[2] RF Patent 2387844 for an invention. Int. Cl. F01G1/077, F02G1/044. Rotary-vane engine with an external supply of heat Y. N. Lukyanov, Y. N. Zhuravlev et al. Publ. 27.04.2010. Bull. Number 12.

[3] RF Patent 2619391 for an invention. Int. Cl. F01C1/44. Rotary Expantion Machine / Y. N. Lukyanov, Y. N. Zhuravlev et al. Publ. 15.05.2017.

[4] Perminov A., Lukyanov Y., Ilyin A., Zhuravlev Y., Tikhonov S., Determination of chamber and piston geometry for a rotary type expansion machine. "Environment. Technology. Resources. Proceedings of the 12th International Scientific and Practical $\begin{array}{llll}\text { Conference", } & \text { 2019, } & 3 \text {, p. } & \text { 197-200, }\end{array}$ https://doi.org/10.17770/etr2019vol3.4060

[5] Zhuravlev Y., Perminov A., Lukyanov Y., Tikhonov S., Ilyin A., Construction of piston outer profile for rotary type expansion machine. "Environment. Technology. Resources. Proceedings of the 12th International Scientific and Practical Conference”, 2019, 3, p. 257-260, https://doi.org/10.17770/etr2019vol3.4059

[6] Y. N. Zhuravlev, I. V. Plokhov, Y. N. Lukyanov, and others, Calculation and Design of Rotaty-blade Machine with External Heat Supply. Monogrphy. Pskov, 2012.

[7] Y. N. Zhuravlev, I. V. Plokhov, Y. N. Lukyanov, and others, Mathematical Models of Physical Processes in Rotary-blade Engine with External Heat Supply. Monogrphy. Pskov, 2012.

[8] S. Semyonov, S. Tikhonov, Y. Lukyanov, A. Perminov, Analysis method of calculation parameters of heat and mass transfer processes in the stirling engine. "Environment. Technology. Resources. Proceedings of the 11th International Scientific and Practical Conference”, 2017, 3, p. 298-303, https://doi.org/10.17770/etr2017vol3.2513

[9] S. Semyonov, S. Tikhonov, Y. Lukyanov, A. Perminov, Optimization of mechanical strength of rotary-vane engine. "Environment. Technology. Resources. Proceedings of the 11th International Scientific and Practical Conference”, 2017, 3, p. 357360, https://doi.org/10.17770/etr2017vol3.2511

[10] Perminov A., Lukyanov Y., Tikhonov S., Ilyin A. Thermodynamic cycle with two-component working fluid. "Environment Technology. Resources. Proceedings of the 11th International Scientific and Practical Conference”, 2015, https://doi.org/10.17770/etr2015vol2.261

[11] Y. N. Zhuravlev, A. L. Perminov, Y. N. Lukyanov, S. I. Tikhonov, S. N. Semenov, Minimization of Mechanical Strength of Rotaryblade Heat Engine with Cam-and-lever Motion Transformer. Bulletin of Pskov State University. Series: Engineering scinces, 2016. 